\title{
Prevalencia de infarto agudo de miocardio en pacientes jóvenes y su patrón angiográfico
}

\author{
Francisco Hernández-Pérez ${ }^{1 *}$ y Alexis D. Núñez-Granados ${ }^{2}$ \\ ${ }^{1}$ Centro de Investigación Educativa y Formación Docente, Centro Médico Nacional Siglo XXI, Instituto Mexicano del Seguro Social; ${ }^{2}$ Servicio de \\ Urgencias, Hospital de Especialidades Dr. Belisario Domínguez, Secretaría de Salud. Ciudad de México, México
}

\begin{abstract}
Resumen
Introducción: El infarto agudo de miocardio en menores de 45 años tiene una baja prevalencia y produce menos daños en las arterias coronarias. Objetivo: Identificar la prevalencia de infarto agudo de miocardio en pacientes menores de 45 años y su patrón angiográfico más frecuente. Método: Estudio transversal en el que se revisaron los expedientes de pacientes con infarto agudo de miocardio menores de 45 años, de donde se extrajeron lo datos generales y los resultados del cateterismo. Se apegó a las normas éticas vigentes. Resultados: Se estudiaron 59 pacientes menores de 45 años con diagnóstico de infarto agudo de miocardio. La media de edad fue de $40.47 \pm 5.0$ años, y hubo 49 hombres y 10 mujeres. La prevalencia global fue del $4.8 \%$. El $44.9 \%$ tenía antecedentes de tabaquismo y el $22.5 \%$ de hipertensión arterial sistémica. De 52 pacientes, 20 (38.4\%) tuvieron lesión solo de una arteria coronaria y 32 (61.5\%) de más de una arteria. Conclusiones: El infarto agudo de miocardio en pacientes menores de 45 años es más común de lo que se piensa, y el tabaquismo es el factor de riesgo más frecuente. El patrón coronario más común fue el mixto, con lesión en más de una arteria, predominando la descendente.
\end{abstract}

Palabras clave: Infarto agudo de miocardio. Joven. Cateterismo cardiaco.

\section{Prevalence of acute myocardial infarction in young patients and angiographic pattern}

\section{Abstract}

Introduction: Acute myocardial infarction in those under 45 years of age has a low prevalence and with less damage to the coronary arteries. Objective: To identify the prevalence of acute myocardial infarction in patients less than 45 years of age as well as the most frequent angiographic pattern. Method: Cross-sectional studies, the files of patients with acute myocardial infarction under 45 years of age were reviewed, from which the general data and the results of catheterization were extracted. We adhered to current ethical standards. Results: 59 patients under 45 years of age with the diagnosis of acute myocardial infarction were studied, the mean age was $40.47 \pm 5.0$ years, 49 were men and 10 women. The overall prevalence was $4.8 \%$. $44.9 \%$ had a history of smoking, followed by $22.5 \%$ who reported suffering from hypertension. From 52 patients, 20 (38.4\%) had only one coronary artery injury and 32 (61.5\%) had more than one artery injury. Conclusions: Acute myocardial infarction in patients under 45 years of age is more common than is thought; smoking was the most common risk factor. The most common coronary pattern was the mixed one, with lesions in more than one artery, predominantly the descending one.

Keywords: Acute myocardial infarction. Young. Cardiac catheterization.

\section{Correspondencia:}

*Francisco Hernández-Pérez

E-mail: mesias.francisco@gmail.com

Disponible en internet: 14-02-2022

Fecha de recepción: 16-08-2021

Fecha de aceptación: 17-11-2021 DOI: $10.24875 / R E I E .21000093$
Rev Educ Investig Emer. 2022;4(1):21-26 www.medicinadeemergencias.com

2604-6520 @ 2021 Sociedad Mexicana de Medicina de Emergencias, AC. Publicado por Permanyer México SA de CV. Este es un artículo open access bajo la licencia CC BY-NC-ND (http://creativecommons.org/licenses/by-nc-nd/4.0/). 


\section{Introducción}

A pesar de que en muchos países la incidencia anual de infarto agudo de miocardio ha ido en descenso, en México, por el contrario, no se ha percibido tal disminución y hay datos que sugieren un incremento. El Instituto Nacional de Estadística y Geografía informó una muerte por cardiopatía isquémica cada 4.3 minutos, siendo el primer motivo de consulta subsecuente en medicina familiar (más de 16.6 millones) y la primera causa de años de vida saludable perdidos por muerte prematura y discapacidad. Tan solo en el Instituto Mexicano del Seguro Social, que cubre a dos terceras partes de la población total de México (> 70 millones), fallecían 138 derechohabientes al día por infarto de miocardio hasta antes de la estrategia "Código infarto». La mortalidad reportada por esta causa según la Organización para la Cooperación y el Desarrollo Económicos era encabezada por México, con un $28 \%$, frente al $7.9 \%$ del promedio ${ }^{1}$, pero el daño no queda ahí, pues luego de un infarto agudo de miocardio la posibilidad de muerte o discapacidad se incrementa 1,5 a 15 veces respecto de la población general. El $18 \%$ al $35 \%$ de los pacientes podrán sufrir un nuevo episodio, aproximadamente el $7 \%$ corren el riesgo de presentar muerte súbita y el $8 \%$ al $11 \%$ podrán padecer un episodio de accidente vascular cerebral2,3. Por otro lado, el infarto agudo de miocardio a edad temprana (< 45 años) se caracteriza por bajas tasas de mortalidad, menos daños extensos de las arterias coronarias, una buena función ventricular izquierda y un pronóstico favorable, y epidemiológicamente los estudios han demostrado diferencias significativas de los perfiles de riesgo en los pacientes jóvenes en comparación con los pacientes mayores ${ }^{4-6}$. Sin embargo, la mayoría de los pacientes jóvenes que sufren un infarto de miocardio tienen al menos una factor de riesgo cardiovascular, y se informa una mayor prevalencia de tabaquismo, antecedentes familiares de prematuridad con cardiopatías congénitas y sexo masculino. De estos factores de riesgo, se ha sugerido que fumar puede ser el más importante de los factores modificables en los pacientes jóvenes, ya que en la mayoría de los estudios realizados en pacientes jóvenes este factor de riesgo es el más persistente de manera independiente ${ }^{7-9}$.

Es a partir de los 35 años de edad que la enfermedad coronaria empieza a cobrar poco a poco protagonismo, y aunque estas enfermedades son la manifestación clínica de la evolución de la aterosclerosis, el proceso comienza a desarrollarse en etapas tempranas de la vida y progresa, de forma asintomática, hasta la adultez, cuando alcanza su manifestación clínica, habitualmente después de la tercera o la cuarta década de la vida. En la adolescencia y el principio de la adultez se adquieren ciertos hábitos (tipo de dieta, sedentarismo, tabaquismo) que luego son difíciles de modificar y predisponen a la enfermedad cardiovascular durante años posteriores; sin embargo, la enfermedad de las arterias coronarias no fue reconocida como común entre pacientes jóvenes (menores de 45 años) hasta el estudio realizado por Yater en $1948^{10,11}$. Ahora bien, la prevalencia de síndrome coronario agudo en estos individuos es variable según la población estudiada, oscilando entre un $2 \%$ y un $10 \%$ de todos los casos de síndrome coronario agudo hospitalizados, y se muestra como una enfermedad típicamente masculina. Las mujeres parece estar protegidas hasta la menopausia del desarrollo de síndrome coronario agudo, con una incidencia en jóvenes muy baja (del $5 \%$ al $10 \%$ de todos los pacientes con infarto de miocardio menores de 45 años) $)^{12}$.

En este grupo de pacientes se presenta un perfil vascular particular: el infarto agudo de miocardio en ausencia de ateroesclerosis es poco común en los mayores de 45 años, pero es responsable de hasta el $20 \%$ de los casos en pacientes jóvenes y suele relacionarse con mayor frecuencia con fenómenos de hipercoagulabilidad, vasculitis, vasoespasmo endógeno o vasoespasmo mediado por sustancias tóxicas; esto subestimando o sin considerar los factores de riesgo principales para la enfermedad coronaria como génesis directa de causalidad en esta población ${ }^{13}$.

Finalmente, habrá que aclarar que no hay un acuerdo claro en cuanto a la edad mínima para ser llamado infarto agudo de miocardio en paciente joven; varios autores indican 50 o 40 años, e incluso con diferencia entre sexos. Para el presente trabajo hemos determinado 45 años como edad límite superior, dada la mayor evidencia y que tener más de 45 años se considera un factor de riesgo mayor para el desarrollo de infarto agudo de miocardio.

\section{Método}

Se trata de un estudio transversal en el que se revisaron todos los expedientes de pacientes con infarto agudo de miocardio menores de 45 años durante el periodo de enero de 2017 a junio de 2020, derivado de la presencia de la pandemia de COVID-19. Se extrajeron los datos generales y con intención los resultados del cateterismo y del procedimiento invasivo. El estudio se llevó a cabo en el Hospital de Especialidades 
Dr. Belisario Domínguez, de la Secretaría de Salud de la Ciudad de México, que cuenta con servicio de cardiología intervencionista, pero que a partir de marzo de 2020 se convirtió en «hospital 100\% COVID» y por ello se suspendieron todos los procedimientos. No se excluyó ni elimino a ningún paciente, dado que todos los menores de 45 años fueron sometidos a cateterismo cardiaco.

Se observaron todas las consideraciones éticas y el estudio fue aprobado por el comité local de ética e investigación.

\section{Análisis estadístico}

Se reportan medias y porcentajes, y se usó la prueba de $\chi^{2}$ para comparar porcentajes.

\section{Resultados}

La prevalencia global fue del $4.8 \%$, con una incidencia del $1.1 \%$ anual de nuevos casos por año por cada 1000 infartos. De un total de 1221 casos de infarto agudo de miocardio, se estudiaron 59 expedientes de pacientes menores de 45 años con dicho diagnóstico. La media de edad fue de $40.47 \pm 5.0$ años, y 49 fueron hombres y 10 mujeres. El $44.9 \%$ tenía antecedentes de tabaquismo (edad promedio de inicio a los $14.94 \pm$ 5.5 años) y el $22.5 \%$ refirió padecer hipertensión arterial sistémica; solo nueve pacientes negaron algún factor de riesgo cardiovascular mayor para cardiopatía isquémica. Así mismo, el cuadro más referido fue el de dolor opresivo retroesternal y solo el $20 \%$ fueron trombolizados (Tabla 1).

\section{Cateterismo}

Se realizó cateterismo a los 59 pacientes jóvenes, de los cuales $6(10.1 \%)$ fueron reportados sin lesión y $1(1.6 \%)$ como procedimiento fallido. De los 52 pacientes restantes, $20(38.4 \%)$ tuvieron lesión solo de una arteria coronaria y $32(61.5 \%)$ de más de una arteria. La arteria lesionada más habitual fue la descendente anterior, con 37 casos $(71.1 \%)$, seguida de la coronaria derecha con 30 casos (57.6\%). Las combinaciones se describen en la tabla 2. No hubo ningún fallecimiento posterior al cateterismo.

El tipo de cateterismo fue en su mayoría primario, en 24 pacientes $(40.7 \%)$ seguido de diagnóstico en $21(35.6 \%) ;$ en el resto no se contó con esa información. El grado de lesión se describe en la tabla 2. De los 52 pacientes con lesión vascular, a 16 (30.7\%) se
Tabla 1. Características generales de los participantes en el estudio

\begin{tabular}{|c|c|}
\hline Característica & $n=59$ \\
\hline Edad (años), media $\pm \mathrm{DE}$ & $40.47 \pm 5.0$ \\
\hline $\begin{array}{c}\text { Sexo, n (\%) } \\
\text { Hombre } \\
\text { Mujer }\end{array}$ & $\begin{array}{l}49(83.1) \\
10(16.9)\end{array}$ \\
\hline $\begin{array}{l}\text { Antecedentes, n (\%) } \\
\text { Tabaquismo } \\
\text { Hipertensión arterial } \\
\text { Diabetes mellitus } \\
\text { Dislipidemia } \\
\text { Infarto previo } \\
\text { Uso de drogas } \\
\text { Más de uno o más FRCV }\end{array}$ & $\begin{array}{c}40(67.8) \\
20(33.9) \\
18(30.5) \\
8(13.6) \\
14(23.7) \\
5(8.5) \\
50(84.7)\end{array}$ \\
\hline $\mathrm{IMC}$, media $\pm \mathrm{DE}$ & $29.22 \pm 5.0$ \\
\hline $\begin{array}{l}\text { Características del dolor } \\
\text { Tórax anterior, opresivo, } \mathrm{n}(\%) \\
\text { Al esfuerzo } \\
\text { Intensidad, media } \pm \mathrm{DE} \\
\text { ACME (min), media } \pm \mathrm{DE}\end{array}$ & $\begin{array}{c}51(86.4) \\
13(26) \\
8.25 \pm 1.5 \\
45.91 \pm 5.3\end{array}$ \\
\hline Uso de trombolítico, n (\%) & $12(20.3)$ \\
\hline $\begin{array}{l}\text { Cara de ECG afectada, n (\%) } \\
\text { Inferior } \\
\text { Anterior } \\
\text { Inferior y lateral }\end{array}$ & $\begin{array}{c}18(46.2) \\
17(43.3) \\
4(10.3)\end{array}$ \\
\hline
\end{tabular}

ÁCME: el punto más alto del dolor; ECG: electrocardiograma; FRCV: factor de riesgo cardiovascular mayor; IMC: índice de masa corporal.

les colocó un solo stent y al resto se les colocó más de uno.

\section{Análisis inferencial}

Al comparar la frecuencia de comorbilidad no se encontró diferencia significativa entre los antecedentes de tabaquismo, dislipidemia o hipertensión y el sexo $(p=0.170, p=0.591$ y $p=0.206$, respectivamente); solo el antecedente de diabetes mellitus fue más común en los hombres, siendo estadísticamente significativo ( $p=0.036)$.

En cuanto a la arteria coronaria más afectada y el sexo, la descendente anterior fue la más frecuentemente lesionada en el sexo masculino ( $p=0.025)$; en el resto de las ramas no hubo diferencia significativa $(p<0.05)$ (Tabla 2 y Figs. 1 a 3 ).

\section{Discusión}

En nuestro estudio, la prevalencia global de infarto agudo de miocardio en pacientes menores de 45 años fue del $4.8 \%$, con una incidencia de un $1.1 \%$ anual de 
Tabla 2. Reporte y topografía de la lesión coronaria

\begin{tabular}{|c|c|}
\hline Tipo de cateterismo & n $(\%)$ \\
\hline $\begin{array}{l}\text { Diagnóstico } \\
\text { Primario } \\
\text { De rescate } \\
\text { Postrombólisis }\end{array}$ & $\begin{array}{l}24(40.7) \\
21(35.6) \\
7(11.9) \\
7(11.9)\end{array}$ \\
\hline CI & $\mathrm{n}=39$ \\
\hline $\begin{array}{l}\text { Tronco común de la } \mathrm{Cl} \\
\text { DA sola } \\
D A+C D \\
D A+C x \\
D A+D g \\
D A+D P \\
D A+P L\end{array}$ & $\begin{array}{c}2(5.1) \\
10(25.6) \\
15(38.4) \\
7(17.9) \\
3(7.6) \\
1(2.5) \\
1(2.5)\end{array}$ \\
\hline CD & $\mathbf{n}=\mathbf{2 3}$ \\
\hline $\begin{array}{l}C D \text { sola } \\
C D+C x \\
C D+D P \\
C D+P L \\
C D+M P\end{array}$ & $\begin{array}{l}8(34.7) \\
9(39.1) \\
3(10.3) \\
2(6.8) \\
1(3.4)\end{array}$ \\
\hline Grado de lesión & n (\%) \\
\hline $\begin{array}{l}\text { Lesión del } 100 \% \\
\text { Lesión del } 90-100 \% \\
\text { Lesión del } 70-89 \% \\
\text { Lesión del } 50-69 \% \\
\text { Ectasia } \\
\text { Normal }\end{array}$ & $\begin{array}{c}26(44.0) \\
8(13.5) \\
11(18.6) \\
4(6.77) \\
3(5.08) \\
7(11.8)\end{array}$ \\
\hline
\end{tabular}

nuevos casos por año por cada 1000 infartos. A pesar de que se sospechaba un ascenso de la frecuencia, en la mayoría de los estudios que marcan la prevalencia de infarto en pacientes jóvenes esta es mayor que la nuestra. Morillas et al. ${ }^{11}$, en 10,213 pacientes, reportan una prevalencia del $6,8 \%$, mientras que Méndez et al. ${ }^{5}$ la cifran en el $6.8 \%$ de 613 pacientes menores de 40 años, y Yandrapalli et al. ${ }^{9}$ en un $19,2 \%$ de 1,462,168 pacientes entre 18 y 44 años. Ahora bien, la incidencia fue bastante baja y nuestro único punto de comparación es el estudio de McManus et al. ${ }^{14}$, que durante 30 años encontró una tasa general de incidencia hospitalaria (por 100,000 residentes) de 66 (intervalo de confianza del 95\% [IC95\%]: 63-69). Solo Alonso et al. ${ }^{10}$ tuvieron una prevalencia del $4.6 \%$ en 202 pacientes menores de 45 años. Sin embargo, es pertinente mencionar las diferencias de sus cortes en cuanto a edad límite, lo que afecta claramente las prevalencias. Respecto a la edad en nuestro estudio (40.47 \pm 5.0 años), 49 fueron hombres (83.1\%) y 10 mujeres, lo cual es muy consistente en la mayoría de los estudios, con un predominio claro del sexo masculino $0^{6,10,12,13,15}$.

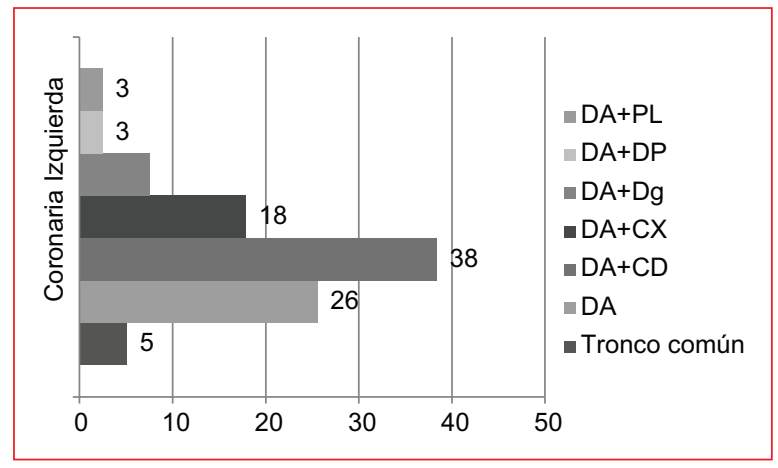

Figura 1. Combinaciones de arterias lesionadas de la coronaria izquierda.

Cx: circunfleja; DA: descendente anterior; Dg: diagonal; DP: descendente posterior; PL: posterolateral.

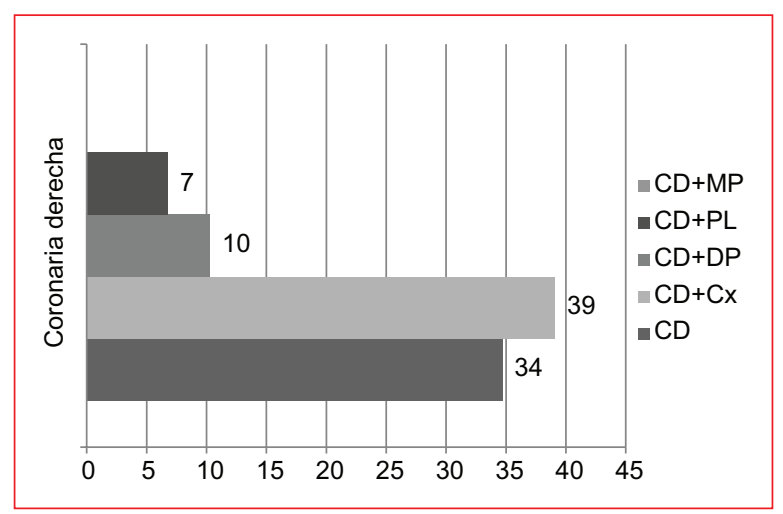

Figura 2. Combinaciones de arterias lesionadas de la coronaria derecha.

CD: coronaria derecha; Cx: circunfleja; DP: descendente posterior; MP: marginal posterior; PL: posterolateral.

En cuanto al resto de los factores de riesgo, el $44.9 \%$ de los pacientes tenían antecedentes de tabaquismo, seguido del $22.5 \%$ que refirieron padecer hipertensión arterial sistémica y un índice de masa corporal (IMC) medio de $29.22 \pm 5.0$ (sobrepeso). Por otro lado, solo nueve pacientes negaron algún factor de riesgo cardiovascular mayor, mismos resultados que reportan la mayoría de los estudios $5,8,13,16$. También en los otros estudios el IMC es alto; en el nuestro predominó el sobrepeso, mayor que en el estudio de Marcen et al..$^{12}$ en población española, en el que el IMC fue de $27.94 \pm$ $4.58 \mathrm{~kg} / \mathrm{m}^{2}$ (17.7-43). Cabe mencionar que el síndrome metabólico se asoció a un peor pronóstico en el estudio de Mathiew et al. ${ }^{17}$ y que incrementó ocho veces las posibilidades de sufrir un infarto agudo de miocardio prematuro (IC95\%: 1.73-39.5), seguido del tabaquismo (razón de momios: 7.76; IC95\%: 1.27-47.3). 


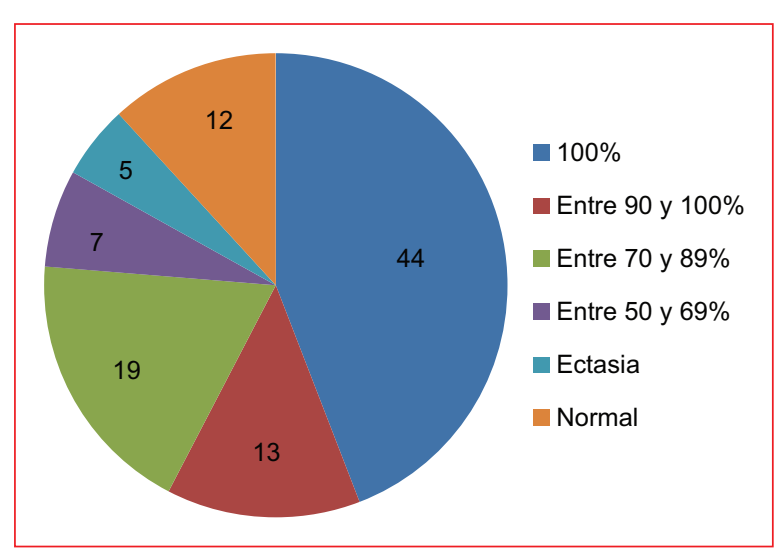

Figura 3. Grado de lesión de la coronaria afectada.

Seis pacientes $(10.1 \%)$ fueron reportados sin lesión coronaria.,es decir, con infarto agudo de miocardio sin enfermedad coronaria aterosclerótica obstructiva(MINOCA) y aunque su fisiopatología no queda clara su prevalencia no es despreciable, ya que hasta el $6 \%$ de todos los síndromes coronarios agudos lo presentan. Los factores de riesgo cardiovascular suelen ser los tradicionales, pero con un incremento de la mortalidad $^{18} \mathrm{y}$, en series recientes, la coronariografía no reveló lesiones coronarias significativas (obstrucción del 50\% o más) en el $1-14 \%$ de los pacientes con infarto agudo de miocardio ${ }^{19}$. Moura et al. ${ }^{20}$ reportan un $1.7 \%$ de coronarias sin lesión entre 1960 casos, y Kang et al. ${ }^{21}$ un $4 \%$ de 8500 casos.

Entre los resultados más relevantes de nuestro estudio, de 52 pacientes, 20 (38.4\%) tuvieron lesión solo de una arteria coronaria y $32(61.5 \%)$ de más de una arteria. La arteria lesionada más común como fue la descendente anterior, con 37 casos $(71.1 \%)$, seguida de la coronaria derecha con 30 casos $(57.6 \%)$. De los 52 pacientes con lesión vascular, a $16(30.7 \%)$ se les colocó un solo stent y al resto se les colocó más de uno. Nuestros resultados son muy semejantes a los de otros estudios, en los que predominan las lesiones multivaso y la descendente anterior es la principal arteria coronaria comprometida, como el estudio de Marcen et al. ${ }^{12}$, que reporta lesión multivaso y multisegmento. El registro nacional de síndromes coronarios agudos en México (RENASICA), en su reporte de 2005, que incluye población general, en 4555 pacientes con infarto de miocardio con elevación del segmento ST la angiografía coronaria mostró enfermedad de la descendente anterior izquierda en el $65 \%$, de la coronaria derecha en el $53 \%$, de la circunfleja en el $37 \%$, del tronco principal izquierdo en el $5 \%$ y oclusión de un injerto venoso en el $1 \% 22$. Aunque la coronaria afectada es muy semejante a lo hallado en nuestro estudio, no así la presencia de varios vasos dañados, como el estudio de Méndez et al. ${ }^{5}$ que identificó la arteria descendente anterior como el vaso culpable del infarto agudo de miocardio en 27 (67,5\%) casos, la coronaria derecha en $8(20 \%)$, la circunfleja en $4(10 \%)$ y el tronco izquierdo en $1(2,5 \%)$, pero en la mayoría $(87,5 \%)$ los pacientes presentaron enfermedad exclusivamente de un vaso, solo $4(10 \%)$ de dos vasos y $1(2,5 \%)$ de tres vasos.

En cuanto a nuestro análisis inferencial, no se pudo demostrar ninguna diferencia significativa entre mujeres y hombres en cuanto a factores de riesgo como tabaquismo, dislipidemia o hipertensión ( $p=0.170$, $p=0.591$ y $p=0.206$, respectivamente); solo el antecedente de diabetes mellitus fue más común en los hombres, siendo estadísticamente significativo ( $p=0.036$ ), lo que es compatible con los resultados de Gupta et al. ${ }^{23}$, que no encuentran diferencias.

Finalmente, del estudio se desprende que, a pesar del pronóstico más favorable en los pacientes menores de 45 años, es muy importante señalar que varios factores de riesgo, como el tabaquismo y el sobrepeso, son factores modificables. En cuanto a la diabetes mellitus y la hipertensión arterial sistémica, son controlables, y de ahí la importancia de estudios como este que manifiestan la relevancia de su control. Además, cabría agregar que, como se observó, aparentemente el daño vascular es peor en nuestros sujetos de investigación.

\section{Conclusiones}

El infarto agudo de miocardio en pacientes jóvenes (menores de 45 años) es más común de lo que se piensa. La comorbilidad más frecuente fue el tabaquismo, la diabetes mellitus y la hipertensión arterial sistémica, y fue escaso el consumo de drogas ilícitas.

El patrón coronario más común fue el mixto, con lesión en más de una arteria, predominado la descendente anterior y la coronaria derecha.

\section{Financiamiento}

La presente investigación no recibió ninguna beca específica de agencias de los sectores público, comercial o sin ánimo de lucro. Todos los recursos fueron aportados por los investigadores. 


\section{Conflicto de intereses}

Los investigadores declaran no tener ningún conflicto de intereses en relación con este artículo.

\section{Responsabilidades éticas}

Protección de personas y animales. Los autores declaran que para esta investigación no se han realizado experimentos en seres humanos ni en animales.

Confidencialidad de los datos. Los autores declaran que han seguido los protocolos de su centro de trabajo sobre la publicación de datos de pacientes.

Derecho a la privacidad y consentimiento informado. Los autores han obtenido el consentimiento informado de los pacientes y/o sujetos referidos en el artículo. Este documento obra en poder del autor de correspondencia.

\section{Bibliografía}

1. Borrayo-Sánchez G, Rosas-Peralta M, Pérez-Rodríguez G, Ramírez-Arias E, Almeida-Gutiérrez E, Arriaga-Dávila JJ. Infarto agudo de miocardio con elevación del segmento ST: Código I. Rev Med Inst Mex Seguro Soc. 2018;56:25-41.

2. Masol O, Trivi M. Infarto agudo de miocardio: estratificación del riesgo isquémico y tratamiento previo al alta. PROSAC. 2009;4:26-42.

3. Castellanos-Rojas R, Ferrer-Herrera I, Segura-Pujal LA, Ojeda-Matías MR, Fernández-Romero D. Infarto agudo del miocardio en pacientes jóvenes. Rev Arch Med Camagüey. 2014;18:667-79.

4. Brscic E, Bergerone S, Gagnor A, Colajanni E, Matullo G, Scaglione L, et al. Acute myocardial infarction in young adults: prognostic role of angiotensin-converting enzyme, angiotensin II type I receptor, apolipoprotein $E$, endothelial constitutive nitric oxide synthase, and glycoprotein IIIa genetic polymorphisms at medium-term follow-up. Am Heart J. 2000;139:979-84.

5. Méndez M, Martínez G, Veas N, Pérez O, Lindefjeld D, Winter JL, et al. Infarto agudo al miocardio en pacientes menores de 40 años. Características clínicas, angiográficas y alternativas terapéuticas. Rev Chil Cardiol. 2013;32:21-7.

6. Wittlinger T, Seifert C, Simonis G, Gerlach M, Strasser RH. Prognosis in myocardial infarction of young patients: results of a prospective registry. Int J Cardiol. 2020;300:1-6.
7. Eyben FE, Bech J, Madsen JK, Efsen F. High prevalence of smoking in young patients with acute myocardial infarction. J R Soc Health. 1996;116:153-8.

8. Iyengara SS, Guptab R, Ravic S, Thangamd S, Alexandere T, Manjunathf $\mathrm{CN}$, et al. Premature coronary artery disease in India: coronary artery disease in the young (CADY) registry. Indian Heart J. 2017;69:211-6.

9. Yandrapalli S, Nabors C, Goyal A, Aronow W, Frishman WH. Modifiable risk factors in young adults with first myocardial infarction. JACC. 2019;73:573-84

10. Alonso-Mariño AL, Alonso-Mariño OL, Grau-Ábalos R. Infarto agudo de miocardio en pacientes jóvenes ingresados en cuidados intensivos. Cor Salud 2012;4:20-9.

11. Morillas PJ, Cabadés A, Bertomeu V, Echanove I, Colomina F, Cebrián J, et al. Infarto agudo de miocardio en pacientes menores de 45 años. Rev Esp Cardiol. 2002:55:1124-31.

12. Marcen-Miravete A, Sánchez-Insa E, Molina-Borao I, Lasala-Alastuey M, Rivero-Fernández E, Fuertes-Ferre G, et al. El infarto agudo de miocardio en individuos jóvenes, un problema de incidencia creciente. Revista de la Sociedad Aragonesa de Cardiología. 2017; 19:5-12.

13. Marín F, Ospina LF. Infarto agudo del miocardio en adultos jóvenes menores de 45 años. Rev Colomb Cardiol. 2004;11:193-204.

14. McManus DD, Piacentine SM, Lessard D, Gore JM, Yarzebski J, Spencer FA, et al. Thirty-year (1975 to 2005) trends in the incidence rates, clinical features, treatment practices, and short-term outcomes of patients $<55$ years of age hospitalized with an initial acute myocardial infarction. Am J Cardiol. 2011;108:477-82.

15. Estévez-Rubido Y, Cairo-Sáez G, Quintero-Valdivié I, Pérez-Rodríguez RM, González-López D. Infarto agudo de miocardio en pacientes menores de 50 años en el Hospital Arnaldo Milián Castro. Revista Cubana de Cardiología y Cirugía Cardiovascular. 2018;24:1-15.

16. Mirza AJ, Taha AY, Khdhir BR. Risk factors for acute coronary syndrome in patients below the age of 40 years. Egypt Heart J. 2018;70:233-5.

17. Mathiew-Quiros A, Salinas-Martínez AM, Guzmán de la Garza FJ, Garza-Sagastegui MG, Guzmán-Delgado NE, Palmero-Hinojosa MG, et al. Infarto agudo al miocardio en jóvenes mexicanos asociado a síndrome metabólico. Gac Med Mex. 2017;153:297-304.

18. Matsudo M, Aladio JM, Swieszkowski SP, Pérez de la Hoz RA. MINOCA, infarto con coronarias normales: ¿la caída del paradigma? Medicina. 2019;79:201-4.

19. Ache $Y$, Guamán $C$, Viñole L, Vignolo $G$. Infarto agudo de miocardio sin lesiones coronarias obstructivas - MINOCA: un enigma para el cardiólogo clínico. Rev Urug Cardiol. 2020;35:77-86.

20. Moura-Schmidt M, Schaan de Quadros A, Schütz-Martinelli E, Mascia-Gottschall CA. Prevalencia, etiología y características de los pacientes con infarto agudo de miocardio tipo 2. Rev Bras Cardiol Invasiva. 2015;23:119-23.

21. Kang WY, Jeong MH, Ahn YK, Kim JH, Chae SC, Kim YJ, et al.; Korea Acute Myocardial Infarction Registry Investigators. Are patients with angiographically near-normal coronary arteries who present as acute myocardial infarction actually safe? Int J Cardiol. 2011;146:207-12.

22. García-Castillo A, Jerjes-Sánchez C, Martínez-Bermúdez $P$, Azpiri-López JR, Autrey-Caballero A, Martínez-Sánchez C, et al. RENASICA II: Registro Mexicano de Síndromes Coronarios Agudos. Arch Cardiol Mex. 2005;75 (Supl 1):S6-S19.

23. Gupta A, Wang Y, Spertus JA, Geda M, Lorenze N, Nkonde-Price C, et al. Trends in acute myocardial infarction in young patients and differences by sex and race, 2001 to 2010. J Am Coll Cardiol. 2014;64:337-45. 